\title{
Una aproximación a la fenomenología de la enfermedad
}

\author{
Carlos Alva-Espinosa
}

Consulta externa de Cardiología, Hospital Ángeles del Pedregal, Ciudad de México, México

\section{Resumen}

La medicina moderna ha experimentado un progreso tecnológico sin duda con mejores resultados, pero la relación médico-paciente se ha socavado en su aspecto más importante, el contacto humano. El enfermo experimenta y enfrenta en soledad su experiencia adversa. Esta experiencia es compleja; no solo son los síntomas de la enfermedad, también comprende la invalidez, el aislamiento, el temor, la dependencia y en ocasiones la culpa. La fenomenología otorga una importancia capital a los datos en la conciencia del enfermo, como hechos dados con valor absoluto. El propósito de este trabajo es profundizar en la experiencia del individuo adulto sano que enferma. La mejor comprensión de esta fenomenología del enfermo por parte del médico y del personal de salud puede favorecer una mejor eficiencia, empatía y compasión en su atención.

PALABRAS CLAVE: Fenomenología. Enfermedad. Experiencia. Síntomas.

\begin{abstract}
Modern medicine has undergone technological progress, undoubtedly with better results, but the doctor-patient relationship has been undermined in its most important aspect, human contact. The patient experiences and confronts in solitude his adverse experience. This experience is complex, not only are the symptoms of the disease, they also include disability, isolation, fear, dependence and sometimes guilt. Phenomenology attaches a paramount importance to the data in the consciousness of the patient, as facts given with absolute value. The purpose of this paper is to deepen the experience of the healthy adult individual who is ill. The better understanding of this phenomenology of the patient by the physician and the health personnel, can favor a better efficiency, empathy and compassion in their care.
\end{abstract}

KEY WORDS: Phenomenology. Disease. Experience. Symptoms.

Correspondencia:

Gac Med Mex. 2017;153:641-644

Contents available at PubMed www.gacetamedicademexico.com 


\section{Introducción}

Todos los pacientes aquejados de una enfermedad con síntomas, que son la mayoría (existen algunas enfermedades o etapas de enfermedad en las que los enfermos no experimentan síntomas; por ejemplo, algunos enfermos con hipertensión arterial sistémica cursan asintomáticos), tienen, de acuerdo con Lifhsitz ${ }^{1}$, algún tipo de las siguientes vivencias: dolor, fiebre, debilidad, opresión, mareo, vértigo, angustia o falta de aire; pero además experimentan en mayor 0 menor grado invalidez, y pueden también percibir una amenaza sobre su persona. Dado que la enfermedad puede complicarse, la amenaza de morir a causa de la enfermedad está presente. En el que padece, la conciencia está en el síntoma, por ejemplo en la cadera dolorosa, lo que Pedro Laín Entralgo ${ }^{2}$ denomina "succión del cuerpo", en nuestro ejemplo "succionado por el dolor en la cadera». Adicionalmente, algunos, de acuerdo con Hernández Bayona ${ }^{3}$, experimentan culpa y necesidad de expiación. Se sienten responsables de lo que les está pasando; por ejemplo, el fumador crónico ante su cáncer de pulmón.

En suma, junto con Estrada ${ }^{4}$ podemos hablar de la pesadez del sufrimiento en el individuo asolado por la enfermedad.

La medicina moderna ha experimentado un gran progreso tecnológico mediante innumerables máquinas diagnósticas, sin duda con mejores resultados, pero la relación médico-paciente se ha socavado en su aspecto más importante, el contacto humano. El tomógrafo o la resonancia magnética se interponen entre el médico y su enfermo. El médico ordena un estudio especializado después de un breve interrogatorio, y en la siguiente consulta, ya con los resultados de los estudios, explicará lo que está pasando; mientras tanto, el paciente continúa la experiencia solitaria de su enfermedad, y ahora con el miedo y la zozobra por lo que le pueda pasar durante el estudio. En aras de la eficiencia tecnológica, se tiende a olvidar las vivencias del enfermo. Un abordaje fenomenológico de la enfermedad podría ayudar a recuperar la importancia de la relación médico-paciente.

\section{Enfoque}

La experiencia de la enfermedad involucra la íntima relación entre el cuerpo y la conciencia, del significado otorgado al síntoma y de su relación con el sujeto que lo experimenta y el mundo que lo rodea. Nada es más misterioso, para el hombre, que el espesor de su propio cuerpo ${ }^{5}$, particularmente cuando este enferma.

Es necesario un abordaje fenomenológico, entendido como el análisis de las vivencias del enfermo en su conciencia. Estos contenidos, como están dados en la conciencia, de acuerdo con Husserl ${ }^{6,7}$ no solo son reales e intencionales, sino que también son contenidos absolutos; en otras palabras, son fenómenos puros de la conciencia, que veremos con mayor detalle más adelante.

El propósito, entonces, de este trabajo es profundizar en la experiencia fenomenológica del individuo adulto sano que enferma, para obtener una mejor comprensión de lo que él vive y con ello fortalecer en la intersubjetividad la comunicación útil y la empatía en la relación del profesional de la medicina con el enfermo.

En la revisión de la literatura sobre fenomenología y enfermedad existen diversos artículos relacionados, pero la mayoría de ellos con enfermedades mentales $^{8-14}$. En este trabajo únicamente serán consideradas las situaciones en que el individuo adulto conserva sus facultades mentales íntegras y padece graves síntomas no psiquiátricos.

\section{Cómo aproximarse fenomenológicamente a la enfermedad}

Un requisito previo a la aproximación fenomenológica de la enfermedad es adentrarnos a la idea de fenómeno. "Fenómeno" define la vivencia del yo en su conciencia, y nada más, pero nada menos ${ }^{7}$. Esta vivencia es compleja y sus contenidos pueden originarse en el exterior del sujeto, en el mundo real (por ejemplo, percibir la sala de operaciones con todos sus componentes, incluyendo el cirujano), o en su interior (digamos sus pensamientos y emociones). En el devenir de la conciencia, los fenómenos generalmente incluyen ambas fuentes: internas y externas. De cualquier manera, el enfoque en este artículo se dirige a lo que está dado en la conciencia del enfermo; lo que él vive debe ser considerado como lo que es para él mismo, como un hecho verdadero y absoluto, lo cual no significa que exista una correspondencia exacta y verdadera con la realidad exterior.

\section{La mente y el cuerpo}

La experiencia del propio cuerpo nos enseña a arraigar el espacio en la existencia. Ser cuerpo es 
estar anudado a un cierto mundo, vivimos nosotros, y nuestro cuerpo no está, ante todo, en el espacio: es del espacio ${ }^{15}$.

En el ser humano, la conciencia tiene, en condiciones normales, una vinculación estrecha con el cuerpo. Siguiendo a Meralu-Ponty ${ }^{15}$, en la percepción del propio cuerpo y de su interpretación habrá que decir que este se interpreta a sí mismo. Yo no estoy delante de mi cuerpo, estoy en mi cuerpo; o mejor, soy mi cuerpo. En la relación mente-cuerpo, la dicotomía desaparece y puede resumirse, para fines de este trabajo, en lo expresado por Ingold ${ }^{16}$ : el cuerpo y la mente no son dos cosas separadas, sino dos maneras de describir la misma cosa, o mejor dicho, el mismo proceso, es decir, la actividad del organismo-persona en su entorno.

El individuo enfermo se enfrenta con sus síntomas como lo hace ante cualquier adversidad: como un todo, mediante un sistema en desarrollo ${ }^{16}$; es decir, participa con su cuerpo, su mente y su bagaje sociocultural, con el que cuenta en el momento de la enfermedad, pero -y esto es muy importante- a partir del inicio del síntoma el individuo reacciona sistémicamente elaborando y creando una defensa con lo que él es, con las herramientas que en ese momento posee, generando modificaciones en su ser en interacción con su padecimiento, esto es, con él mismo, en ese presente con el que está viviendo y que parece dilatarse. Aunque esta defensa es plástica y se adecúa a la nueva situación, generalmente no tiene éxito o este es parcial, en términos de restablecer el equilibrio (homeostasis), y tiende a replegarse. En qué consiste este repliegue es lo que revisaremos a continuación.

\section{¿Qué es lo esencial en la fenomenología de la enfermedad?}

Lo esencial de la fenomenología de la enfermedad es el aislamiento, el repliegue del ser y la soledad. Cuando el entorno es la enfermedad del propio cuerpo, el vínculo de la conciencia con el cuerpo se agudiza. Si en condiciones normales sabemos dónde están las partes de nuestro cuerpo que nos acompañan, pero solo de manera marginal, y podemos, por ejemplo, conducir el auto pensando en el plan del día, en otras palabras, la conciencia está en otra parte, sí dirige al mundo fuera del cuerpo y sobre él actúa. La conciencia siempre es conciencia de algo, y en la enfermedad la conciencia se dirige al cuerpo, específicamente se repliega hacia la parte «doliente» del mismo. Se produce un robo de atención de la conciencia, que no va al exterior y solo se atiende al mundo más inmediato: el propio cuerpo. Es la existencia del yo experimentando la soledad con su cuerpo, que es también él mismo. Es la experiencia de la cárcel en la propia conciencia. Es incapaz de salir al mundo porque se encuentra rodeado por las barreras dolientes del cuerpo que le reclaman su total atención, pero el cuerpo, en combinación con la mente, no es una celda simple, sino que es todo un nudo de significaciones vivientes, pasadas, presentes y futuras que elaboran una reorganización adaptativa a la nueva situación.

\section{Limitación o pérdida de libertad de la conciencia}

No se puede ser libre cuando la voluntad y la conciencia se encuentran «secuestradas» por el fenómeno del dolor o la disnea. La conciencia toda ella está ocupada por el dolor, el sitio del dolor y sus repercusiones emocionales y cognitivas. Si acaso, el pensamiento y la voluntad son libres por momentos, cuando la intervención del médico es efectiva.

Bajo las sensaciones negativas de experiencia enferma, los pensamientos están acotados y describen una trayectoria circular sobre la vivencia de sentirse mal, de estar enfermo, regresando incesantemente.

\section{Consecuencias}

Desde luego que existe variabilidad en la fenomenología de la enfermedad en los diferentes individuos. Puede variar dependiendo de la estructura de su personalidad, de su historia y de la cultura en la que han estado inmersos. Ingold ${ }^{17}$, sin embargo, indica que es importante, independientemente de las diferencias, identificar y tener presente y conocer lo mejor que podamos los elementos comunes y más trascendentes de la fenomenología del enfermo, para ayudarlo con más eficiencia y sobre todo acompañarlo con más humanidad, en el sentido de auténtica empatía.

Para quien vive la experiencia de estar enfermo, en medio del sufrimiento está la soledad; el paciente vive sus síntomas agudamente solitario, luego amenazado 1 . Ya se ha violentado la integridad de la salud a través de los síntomas, pero junto con experimentarlos se genera la experiencia de una incertidumbre amenazadora: ¿la causa de mi condición, que desconozco, es grave? ¿Progresarán mis molestias o aparecerán otras peores? ¿Podrá complicarse? E incluso 
la amenaza terminal, la amenaza de la nada: ¿puedo morir? Al mismo tiempo se descubre inválido, no puede trabajar y, en casos extremos, es incapaz de asistirse en su cuidado personal y depende del otro. A esta vivencia de invalidez y dependencia puede sumarse la incertidumbre económica y el pensamiento que lleva aparejado: dejar en la indefensión económica a sus familiares.

Finalmente, el enfermo puede atribuirse a sí mismo la responsabilidad de su enfermedad, por ejemplo el haber sido o ser fumador o alcohólico. El fenómeno en la conciencia de la culpa y sus derivados: el autorreproche y la minusvalía pueden rebasar todos los demás y provocar una seria depresión.

\section{Conclusiones}

- La fenomenología otorga una importancia capital a los datos en la conciencia del enfermo, como hechos dados con valor absoluto.

- El enfermo se enfrenta a la adversidad de la enfermedad mediante un sistema en desarrollo con todo lo que cuenta, con un éxito, en el mejor de los casos, parcial.

- El sufrimiento, la soledad, la amenaza, la invalidez, la libertad limitada, la dependencia, y en ocasiones la culpa, hacen de la vivencia de la enfermedad una de las peores de la experiencia humana.
- La mejor comprensión de esta fenomenología del enfermo por parte del médico y del personal de salud puede favorecer una mejor eficiencia, empatía y compasión en su atención.

\section{Bibliografía}

1. Lifhsitz A. La nueva clínica. México D.F.: Conacyt Intersistemas; 2014.

2. Laín Entralgo P. La medicina actual. Madrid: Triacastela; 2011.

3. Hernández Bayona G. Fenomenología de algunos síntomas de la depresión. Universitas Médica. 2007;48:228-40.

4. Estrada DA. Hacia una fenomenología de la enfermedad. latreia. 2012;25:277-86.

5. Le Bretón D. Antropología del cuerpo y modernidad. Buenos Aires: Nueva Visión; 2002. p. 7.

6. Husserl E. La idea de la fenomenología. México D.F.: Fondo de Cultura Económica; 2015. p. 34-56.

7. Husserl E. Investigaciones lógicas II. Madrid: Alianza; 2006. p. 763-77.

8. Cumming TB, Churilov L, Skoog I, et al. Little evidence for different phenomenology in poststroke depression. Acta Psychiatr Scand. 2010;121:424-30.

9. Narbona J. Depressive phenomenology at the outset of neuropaediatric diseases. Rev Neurol. 2014;58(Suppl 1):S 71-5.

10. Vingerhoets AJ, Van Huijgevoort M, Van Heck GL. Leisure sickness: a pilot study on its prevalence, phenomenology, and background. Psychother Psychosom. 2002;71:311-7.

11. Häfner H. Descriptive psychopathology, phenomenology, and the legacy of Karl Jaspers. Dialogues Clin Neurosci. 2015;17:19-29.

12. Matsnev El, Yakovleva IY, Tarasov IK, et al. Space motion sickness: phenomenology, countermeasures, and mechanisms. Aviat Space Environ Med. 1983;54:312-7.

13. Samudra N, Patel N, Womack KB, et al. Psychosis in Parkinson disease: a review of etiology, phenomenology, and management. Drugs Aging. 2016;33:855-63.

14. Zhou YR. The phenomenology of time: lived experiences of people with HIV/AIDS in China. Health (London). 2010;14:310-25.

15. Meralu-Ponty M. Fenomenología de la percepción. Barcelona: Planeta; 1985. p.165-70

16. Ingold T. Tres en uno: cómo disolver las distinciones entre mente, cuerpo y cultura. En: Sánchez Criado T, editor. Tecnogénesis: la construcción técnica de las ecologías humanas. Madrid: AIBR; 2008. p. 1-35.

17. Ingold $\mathrm{T}$. The perception of the enviroment. Londres/NewYork: Routledge; 2000. p. 406-19. 